\title{
Chronic effects of different neuromuscular training on hemodynamic responses estimated vo2max and walking performance in the elderly
}

Fabiana Rodrigues Scartoni ( $\sim$ fabiana.scartoni@ucp.br)

Laboratory of Sport and Exercise Sciences, Catholic University of Petrópolis, RJ, Brazil

\section{Leandro Oliveira Sant'Ana}

Postgraduate Program in Physical Education, Federal University of Juiz de Fora, MG, Brazil.

\section{Jeferson Macedo Vianna}

Postgraduate Program in Physical Education, Federal University of Juiz de Fora, MG, Brazil.

\section{Sérgio Machado}

Neuroscience of Physical Activity, Salgado de Oliveira University, RJ.

\section{Estélio Henrique Martin Dantas}

Stricto Sensu Graduate Program in Nursing and Biosciences - PPgEnfBio, from the Federal University of the State of Rio de Janeiro - UNIRIO, RJ.

\section{Henning Budde}

Faculty of Human Sciences, Medical School Hamburg, University of Applied Science and Medical University, Hamburg 20457

\section{Research Article}

Keywords: cardiovascular, cardiorespiratory, walking performance, RPP, systolic, diastolic

Posted Date: February 15th, 2021

DOl: https://doi.org/10.21203/rs.3.rs-227737/v1

License: (c) (1) This work is licensed under a Creative Commons Attribution 4.0 International License. Read Full License 


\title{
Chronic effects of different neuromuscular training on hemodynamic responses estimated $\mathrm{vo}_{2}$ max and walking performance in the elderly.
}

\author{
Fabiana Rodrigues Scartoni ${ }^{1,2^{*}}$, Leandro de Oliveira Sant'Ana ${ }^{3,4}$, Jeferson Macedo \\ Vianna $^{3,4}$, Sérgio Machado ${ }^{5,6}$, Estélio Henrique Martin Dantas ${ }^{1}$, Henning Budde ${ }^{7}$ \\ ${ }^{1}$ Stricto Sensu Graduate Program in Nursing and Biosciences - PPgEnfBio, from the Federal \\ University of the State of Rio de Janeiro - UNIRIO, Rio de Janeiro, Brazil, \\ ${ }^{2}$ Laboratory of Sport and Exercise Sciences, Catholic University of Petrópolis, RJ, Brazil. \\ ${ }^{3}$ Postgraduate Program in Physical Education, Federal University of Juiz de Fora, MG, Brazil. \\ ${ }^{4}$ Strength Training Studies and Research Laboratory, Federal University of Juiz de Fora, MG, \\ Brazil. \\ ${ }^{5}$ Laboratory of Neuroscience of Physical Activity, Salgado de Oliveira University, RJ, Brazil \\ ${ }^{6}$ Laboratory of Physical Activity Neuroscience, Salgado de Oliveira University, Rio de Janeiro, \\ Brazil \\ ${ }^{7}$ Faculty of Human Sciences, Medical School Hamburg, University of Applied Science and \\ Medical University, Hamburg 20457, Germany \\ * fabiana.scartoni@ucp.br
}

\begin{abstract}
Resistance training is an excellent intervention for obtaining different physiological improvements in the elderly. The present study aimed to investigate the chronic effects of different neuromuscular training on cardiovascular, cardiorespiratory, and walking performance responses in the elderly. One hundred five people (64 women and 41 men) participated in the study, physically active, all over 60 years old. They were randomized to compose three groups: Strength Group, $(n=35)$, Resistance Group $(n=35)$, and Control Group, $(n=35)$. Both experimental groups (Strength Group and resistance group) performed four weeks of adaptation and 12 weeks of specific training. The Strength Group and the resistance group did four weeks adaptation phase and 12 weeks intervention load with $70-85 \%$ and $50-70 \%$, respectively. The same exercise program was used in the experimental groups, using the alternating method by segment with the bench press, squat, one-sided curved stroke, leg extension, biceps curl, knee flexion, Triceps forehead. Both strength and resistance groups carried out two weekly sessions with an interval of at least 48 and a maximum of 72 hours. A pre and post-evaluation of resting heart rate (HR), blood pressure (systolic and diastolic), rate pressure product (RPP) was performed, and oxygen absorption $\left(\mathrm{VO}_{2 \max }\right)$ and walking performance were estimated through the 1600 test meters. In systolic pressure, there was an improvement for the Strength Group $(p=0.007)$ but not for the resistance group $(p=0.172)$ and control group $(p=0.999)$. Between groups, differences were observed between the Strength Group with the control group ( $\mathrm{p}$ $<0.0001)$. For diastolic pressure, there was a difference for the Strength Group $(p=0.000)$ and not for the resistance group ( $\mathrm{p}=0.916)$ and the control group $(\mathrm{p}=0.107)$. in an inter-group comparison, differences were observed for the Strength Group $(p<0.0001)$ and resistance group $(p=0.001)$ when compared with the control group. For heart hate and double product, in the intra-group analysis, no difference was observed ( $>00.05)$, but in the intergroup, for both variables, the difference was observed for the Strength Group and resistance group when compared with the control group $(\mathrm{p}<0.0001)$. Concerning $\mathrm{VO}_{2 \max }$, the resistance group presents better intra and inter-group results $(\mathrm{p}<0.05)$. In walking performance, there were improvements for the Strength Group $(p=0.027)$ and resistance group $(p<0.0001)$. among the groups, the resistance group was the best compared to the Strength Group $(\mathrm{p}=0.000)$ and the control group $(p<0.0001)$. Conclusion resistance training can be a great strategy for hemodynamic and cardiorespiratory improvement in the elderly.
\end{abstract}




\section{Introduction}

The elderly population in the world already expresses a very high statistic, and, in the coming years, it will continue in a significant progression. It is estimated that in 2025 about 800 million individuals in the world population, whether elderly ${ }^{1}$. However, based on this expectation, one of the situations that deserves attention is the aging process's consequences, which are naturally inevitable ${ }^{2}$. Therefore, considering the physiological and functional issues, aging can cause some changes in these capacities behavior and, this can offer reductions (and not losses) of some functionalities ${ }^{3}$.

cardiovascular4, respiratory5, neuromuscular6, neurological7

Regarding the repercussions of aging on the elderly's physiological and functional actions, changes can affect the cardiovascular ${ }^{4}$, cardiorespiratory $^{5}$, neuromuscular ${ }^{6}$, neurological ${ }^{7}$, and other systems, impacting the efficiency of functional capacity and independence of movement of the elderly ${ }^{8}$.

However, physical exercises are an excellent intervention for conditional improvement and help in the aging process ${ }^{4}$. There is evidence that the physiological and functional reductions of the elderly are more related to low physical conditioning than aging itself ${ }^{9}$.

Based on the above, improving the fitness index can offer significant (and positive) responses in several functionalities in the elderly ${ }^{10}$. Therefore, for there to be an increase in physical condition in the middle of the aging process, continuous physical exercise is essential for promoting and maintaining positive responses and obtaining a better quality of life ${ }^{11}$.

Perhaps, one of the most affected systems with aging is neuromuscular, where it determines the muscular capacities, as strength, resistance, and power, considered as primary valences in locomotion with quality, that besides the motor factor, aging can cause, through neuromuscular changes, the process of sarcopenia ${ }^{12}$. However, physical exercises are already well established as the primary intervention for the elderly ${ }^{13}$.

Therefore, some positions direct the importance of some criteria to ensure an efficient and safe practice for this population ${ }^{14}$. Recently, a specific direction for neuromuscular training in the elderly was published, placing on the agenda several essential aspects of this type of activity in the face of the aging process ${ }^{15}$. Other physiological systems deserve complete attention in the elderly, such as cardiovascular and cardiorespiratory, and the importance of physical training is inevitable for better conditions for these functionalities ${ }^{16}$, especially for this population ${ }^{3}$.

However, when referring to cardiovascular and cardiorespiratory behavior in the elderly, studies, in large part, are related to interventions with exercises with aerobic characteristics (performed on treadmills, cycle ergometers, among others) ${ }^{4}$

Concerning studies related to neuromuscular training in cardiovascular and cardiorespiratory responses in the elderly, research is still scarce, even knowing the importance of these types of training for this population ${ }^{13,15}$; and these features integrate essential actions for a greater capacity for functional autonomy ${ }^{8}$, thus enabling the promotion of health and quality of life for this population ${ }^{9}$. Therefore, in this study, we call attention to the variables of muscle strength prescribed at different intensities as an instrument for improving physical conditioning and, consequently, the performance of movement execution, influencing cardiorespiratory variables studied here.

However, the present study aimed to investigate the chronic effects of different neuromuscular training on cardiovascular hemodynamic, cardiorespiratory $\left(\mathrm{VO}_{2 \max }\right.$ estimated), and walking performance. This study anticipates that older people who do strength training will present better hemodynamic responses in the estimated $\mathrm{VO}_{2 \max }$ and 
walking performance.

\section{Results}

All participants performed an anthropometric assessment before and after the intervention; no significant difference was observed $(\mathrm{p}>0.05)$ in any of the variables assessed for the groups investigated (Table 1).

\begin{tabular}{|c|c|c|c|c|c|c|}
\hline \multirow{3}{*}{$\begin{array}{l}\text { Variable } \\
\text { s } \\
\end{array}$} & \multicolumn{2}{|c|}{$\begin{array}{c}\text { Strength Group } \\
\mathrm{N}=35 \text { (69.08 } \pm 5.9 \text { age) }\end{array}$} & \multicolumn{2}{|c|}{$\begin{array}{c}\text { Resistance Group } \\
\mathrm{N}=35 \text { (68.6 } \pm 5.9 \text { age) }\end{array}$} & \multicolumn{2}{|c|}{$\begin{array}{c}\text { Control Group } \\
\mathrm{N}=35(69.4 \pm 8.1 \text { age })\end{array}$} \\
\hline & \multicolumn{2}{|c|}{ Moment } & \multicolumn{2}{|c|}{ Moment } & \multicolumn{2}{|c|}{ Moment } \\
\hline & Pre & Post & Pre & Post & Pre & Post \\
\hline $\begin{array}{l}\text { Weight } \\
\text { (kg) }\end{array}$ & $67.7 \pm 11.1$ & $67.7 \pm 11.1$ & $66.9 \pm 11.9$ & $66.5 \pm 11.7$ & $68.6 \pm 11.1$ & $70.3 \pm 10.6$ \\
\hline $\begin{array}{l}\text { Height } \\
(\mathrm{cm})\end{array}$ & $158.6 \pm 8.9$ & $159.0 \pm 9.1$ & $158.2 \pm 0.08$ & $158.2 \pm 0.08$ & $163.6 \pm 0.07$ & $163.6 \pm 0.07$ \\
\hline $\begin{array}{c}\text { BMI }(\mathrm{kg} / \\
\left.\mathrm{m}^{2}\right)\end{array}$ & $27.03 \pm 4.04$ & $26.8 \pm 4.1$ & $26.6 \pm 3.7$ & $26.6 \pm 3.7$ & $26.08 \pm 4.5$ & $26.4 \pm 4.3$ \\
\hline Fat (\%) & $32.7 \pm 7.7$ & $32.6 \pm 7.8$ & $31.5 \pm 6.9$ & $31.5 \pm 6.9$ & $28.4 \pm 9.4$ & $28.4 \pm 9.4$ \\
\hline
\end{tabular}

Table 1. Anthropometric assessment before and after the intervention, for training and control groups.

An assessment of hemodynamic behavior before and after the intervention was performed (Table 2). For this analysis, systolic blood pressure (SBP) and diastolic blood pressure (DBP), resting heart rate (HR), and rate pressure product (RPP) were used. Comparisons were made between groups (pre and post times) and between groups (post times). A significant interaction was observed for SBP $(p=0.019)$, DBP $(\mathrm{p}<0.0001), \operatorname{HR}(\mathrm{p}=0.049)$ and for RPP ( $\mathrm{p}=0.042)$. In the SBP analysis, in comparison to pre and post intragroup, a significant difference was observed for the Strength Group $(\mathrm{p}=0.007)$ and not for the resistance $(\mathrm{p}=0.172)$ and control groups $(\mathrm{p}=0.999)$. In the inter-group comparison, at the moment after the intervention, there was a significant difference for the Strength Group compared to the control group $(\mathrm{p}<0.0001)$. Regarding BPD, in the pre and post-evaluation, intra groups, a significant improvement of this variable was observed for the Strength Group $(p=0.000)$ and not for the resistance $(p=0.916)$ and control groups $(p=0.107)$. In the inter-group comparison, after the intervention, a significant difference was observed for the strength $(\mathrm{p}<0.0001)$ and resistance $(\mathrm{p}=0.001)$ groups compared with the control group.

Regarding HR, for intra- and post-group comparison, no significant differences were observed for the strength $(p=0.337)$, resistance $(p=0.712)$ and control $(p=0.974)$ groups. After the intervention, there was a significant improvement in the strength and resistance group in the inter-group comparison compared to the control group $(\mathrm{p}<0.0001)$. Finally, the RPP [HR $(\mathrm{bpm}) \times \mathrm{SBP}(\mathrm{mm} / \mathrm{Hg})]$ was calculated and in the pre and post intra-group comparison, no differences were observed for the strength $(p=0.913)$, resistance $(p=0.067)$ and control $(p=0.962)$. Regarding the inter-group comparison, after the intervention, there was a significant improvement in the strength and resistance group compared to the control group $(\mathrm{p}<0.0001)$. Finally, the RPP [HR (bpm) x SBP (mm/Hg)] was calculated and in the pre and post intra-group comparison, no differences were observed for the strength $(p=0.913)$, resistance $(p=0.067)$ and control ( $p=0.962)$. Regarding the inter-group comparison, after the intervention, there was a significant improvement in the strength and resistance group compared to the control group $(p<0.0001)$. 


\begin{tabular}{|c|c|c|c|c|c|c|}
\hline \multirow[t]{3}{*}{ Variables } & \multicolumn{6}{|c|}{ Groups } \\
\hline & \multicolumn{2}{|c|}{$\begin{array}{c}\text { Strength Group } \\
\text { Moment }\end{array}$} & \multicolumn{2}{|c|}{$\begin{array}{c}\text { Resistance Group } \\
\text { Moment }\end{array}$} & \multicolumn{2}{|c|}{$\begin{array}{c}\text { Control Group } \\
\text { Moment }\end{array}$} \\
\hline & Pre & Post & Pre & Post & Pre & Post \\
\hline $\begin{array}{l}\text { Systolic pressure } \\
(\mathrm{mm} / \mathrm{Hg})\end{array}$ & $136.57 \pm 17.14$ & $122.25 \pm 12.83^{* \dagger}$ & $142.28 \pm 18.32$ & $132.85 \pm 14.46$ & $141.14 \pm 17.95$ & $142.57 \pm 18.04$ \\
\hline $\begin{array}{l}\text { Diastolic pressure } \\
(\mathrm{mm} / \mathrm{Hg})\end{array}$ & $85.42 \pm 10.93$ & $76.12 \pm 8.43^{* \dagger}$ & $81.42 \pm 6.01^{\dagger}$ & $79.42 \pm 6.39$ & $82.28 \pm 7.70$ & $87.42 \pm 9.50$ \\
\hline $\begin{array}{l}\text { Heart rate } \\
\text { (bpm) }\end{array}$ & $86.97 \pm 17.07$ & $92.64 \pm 9.69^{\dagger}$ & $97.48 \pm 10.67$ & $93.60 \pm 9.36^{\dagger}$ & $105.82 \pm 10$ & $107.88 \pm 9.48$ \\
\hline $\begin{array}{l}\text { Rate-pressure product } \\
\text { (bpm x mm/Hg) }\end{array}$ & $\begin{array}{c}11862.28 \pm 2658.1 \\
9\end{array}$ & $11312.25 \pm 1604.50^{\dagger}$ & $13886.85 \pm 2459.58$ & $12429.71 \pm 1808.27^{\dagger}$ & $14923.42 \pm 2291.63$ & $14357.71 \pm 2164.50$ \\
\hline
\end{tabular}

Table 2. Hemodynamic assessment before and after the intervention, for training and control groups.

* Significant difference compared to the intra-group pre-intervention moment $(\mathrm{p}<0.05)$

$\dagger$ Significant difference compared with the Control Group for the post-intervention moment $(\mathrm{p}<0.05)$. 
In the $\mathrm{VO}_{2 \max }$ analysis, this measure was calculated in absolute and relative values. In absolute $\mathrm{VO}_{2 \max }$ (Figure 1), there was an interaction between the groups surveyed $(p=0.006)$. In the pre and postintervention analysis, a significant difference was observed for the resistance group $(p=0.002)$, but not for the strength $(p=0.742)$ and control groups $(p=0.270)$. In the post-intervention, there was a significant improvement in the resistance group compared to the strength $(p=0.000)$ and control groups $(p<0.0001)$.

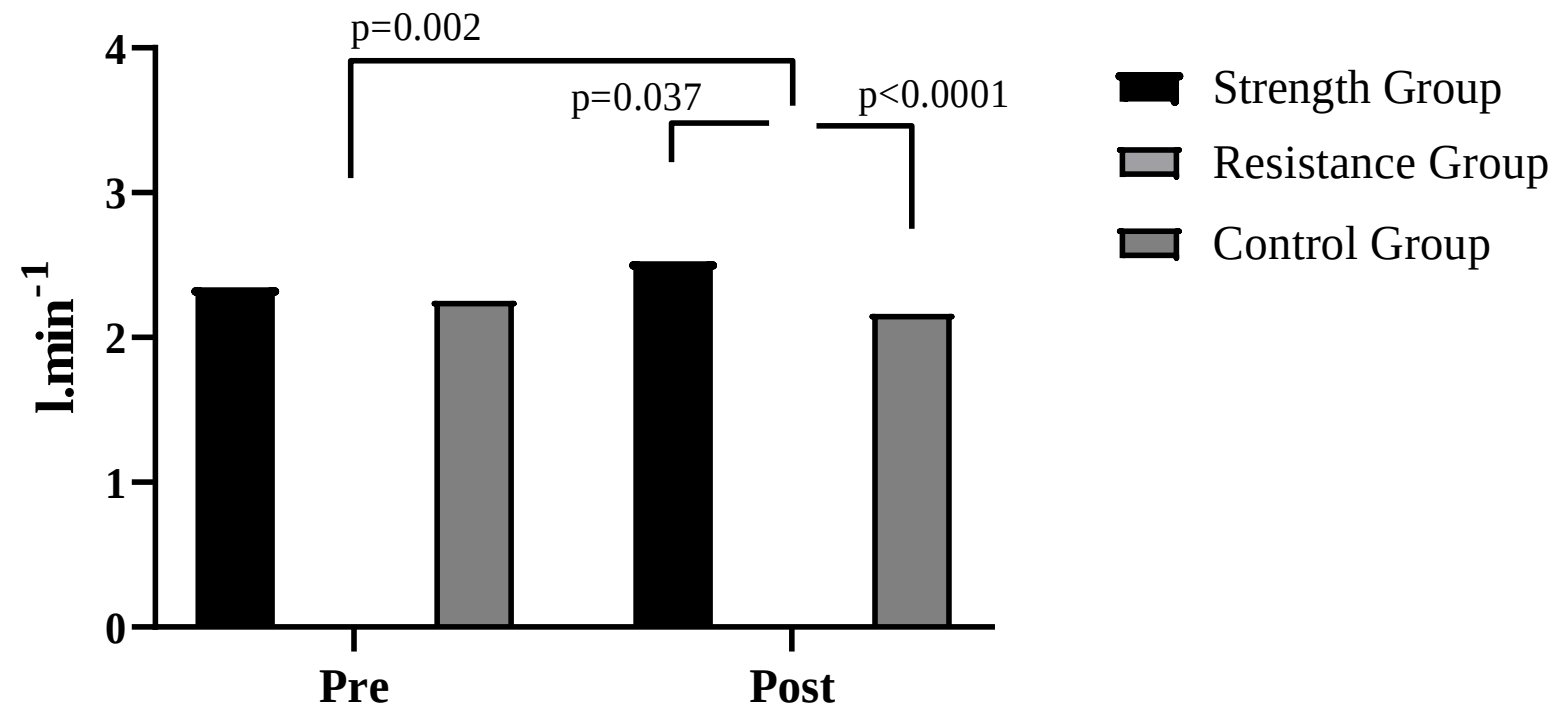

Figure 1. $\mathrm{VO}_{2 \max }$ absolute for the groups, before and after the intervention.

The relative $\mathrm{VO}_{2 \max }$ was also calculated (Figure 4). For this measure, there was an interaction between the groups $(\mathrm{p}<0.0001)$. There was a significant difference for the resistance group $(\mathrm{p}<0.0001)$. In contrast, the strength $(\mathrm{p}>0.9999)$ and control $(\mathrm{p}=0.884)$ groups did not show significant differences. There was a significant difference in the resistance group for the post-intervention inter-group analyses compared to the strength and control groups $(\mathrm{p}<0.0001)$. 


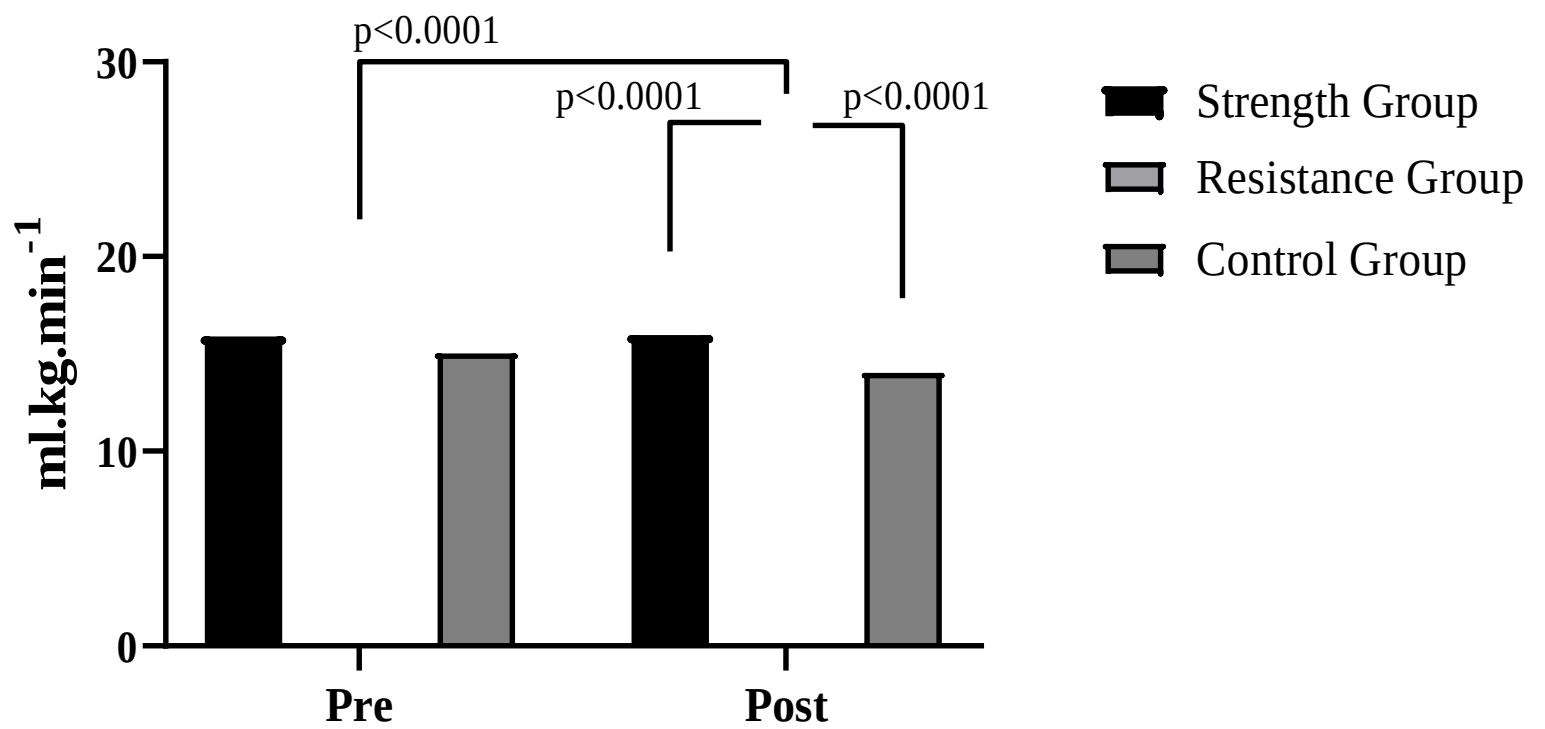

Figure 2. Relative $\mathrm{VO}_{2 \max }$ for the groups, before and after the intervention.

Additionally, the performance of the 1600-meter walk was assessed through the activity time (Figure 3). An interaction was observed between the investigated groups $(\mathrm{p}<0.0001)$. In the pre and post-intervention assessment, a significant difference was observed for the strength $(\mathrm{p}=0.027)$ and resistance $(\mathrm{p}<0.0001)$ groups, but not for the control group $(\mathrm{p}=0.850)$. In the post-intervention inter-group analysis, there was a significant difference in the resistance group compared to the strength $(p=0.000)$ and control $(p<0.0001)$ groups. 


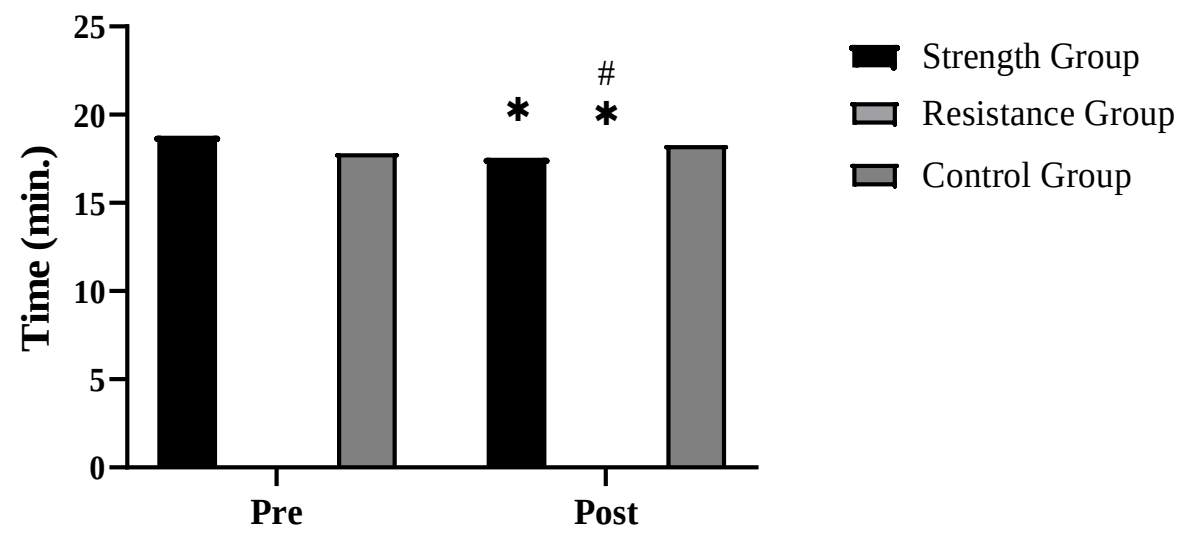

* Significant difference compared to the pre-intervention moment, for the same group $(\mathrm{p}<0.05)$.

\# Significant difference compared to the strength and control groups, after intervention $(\mathrm{p}<0.05)$.

Figure 3. Walking performance for groups, before and after interventions.

\section{Discussion}

The present study aimed to investigate the chronic effects of different neuromuscular training (strength and endurance) on hemodynamic responses, estimated $\mathrm{VO}_{2 \max }$, and walking performance in physically active older people present in physical activities equal to or more than 150 minutes weekly. Both experimental interventions enabled, in some way, positive results in individuals.

The hemodynamic evaluation was performed using blood pressure variables (SBP and DBP), resting heart rate (HR), and rate pressure product (RPP). In intra-group analyzes (pre and post), individuals who performed strength training (strength group ) obtained better results for SBP $(p=0.007)$. Those who did resistance training (resistance group) were unable to achieve significant responses ( $\mathrm{p}=$ 0.172 ), as well as those who did not perform an exercise (control group) ( $p=0.999)$. For post-intervention analysis between groups, significant differences were observed in the strength group vs. control group comparison $(\mathrm{p}<0.0001)$, with the strength group showing the best result. In the same way, it went on to the DBP analyzes, were those who performed strength work significantly improved $(\mathrm{p}=0.000)$, while individuals who performed resistance training $(p=0.916)$ and those who participated in the control group $(p=0.107)$ did not improve this variable, but neither did it worsen. In the post intergroup comparison, there were differences in the strength group vs. control group $(\mathrm{p}<0.0001)$ and resistance group vs. control group ( $\mathrm{p}=0.001)$ comparisons, with the experimental groups showing better results.

For HR, no significant differences were observed in the pre and post intra moments in any group ( $p>0.05)$. However, significant differences were observed when comparing the strength group vs. control group and resistance group vs. control group after the intervention ( $p<0.0001)$. Finally, in the RPP evaluation, in the pre and post analyzes, no significant differences were observed for strength group ( $\mathrm{p}=$ 0.913), resistance group $(\mathrm{p}=0.067)$ and control group $(\mathrm{p}=0.962)$. However, after the inter-group analysis, significant differences were observed for the strength group vs. control group and resistance group vs. control group ( $\mathrm{p}<0.0001)$.

These findings corroborate those of $\mathrm{Mota}^{17}$; for four months, progressive load training and regressive repetitions were performed in hypertensive older women and, they obtained significant improvements in SBP (less $14 \mathrm{~mm} / \mathrm{Hg}$ ) and DBP (less $3.6 \mathrm{~mm} / \mathrm{Hg})(\mathrm{p}<0.05)$. In the present study, in the strength group, a reduction of $14.3 \mathrm{~mm} / \mathrm{Hg}$ and $9.3 \mathrm{~mm} / \mathrm{Hg}$ was observed for SBP and DBP, respectively. However, in the study mentioned above ${ }^{17}$, there was a progression (for each month of intervention) of loads (60 to $80 \%$ of $1 \mathrm{RM}$ ) and regression in the volume of repetitions (12 to 8 repetitions), being that acutely (when individuals performed 12 repeats) and chronic (when individuals performed eight repetitions), significant differences were observed. In the present study, linear training was carried out, 
where significant differences were observed (for SBP and DBP) for those who performed fewer repetitions and more loads.

Additionally, to corroborate the present study's findings, other studies have also intervened with resistance training and obtained favorable results in hemodynamic responses. Da Cunha ${ }^{18}$ underwent 14week training and, after the intervention, improved the SBP $(p=0.001)$ and DBP $(p=0.008)$ of the elderly. In this sense, 10-week training with resistance exercises reduced the SBP of hypertensive and normotensive elderly individuals ${ }^{19}$. Our findings demonstrated that the applied interventions were insufficient to promote significant improvements (reductions) in this variable regarding HR. However, these results are in line with other studies that found insignificance between different HR modalities, which proves to be a variable of more discrete (positive) changes ${ }^{4}$.

$\mathrm{VO}_{2 \max }$ was estimated through the 1600 meter test. For the elderly, these tests are more appropriate than the maximum tests themselves due to the functional capacity of the elderly, which are often restricted $^{20}$. And, it seems that resistance training is the most plausible to obtain improvements to this capacity. Studies related to resistance training and oxygen consumption are scarce, and, therefore, it becomes more difficult to delineate certain conclusions about this. However, other studies have shown that resistance training (80\% $1 \mathrm{RM})$ alters autonomic cardiac functions, maximum oxygen consumption after exercise, but not $\mathrm{VO}_{2 \max }$ kinetics of young individuals, influencing chronic changes ${ }^{21}$.

However, in the present study, it was observed that those who did the training using the longest tension time (resistance) improved significantly $(\mathrm{p}<0.05)$ both in the analysis of absolute and relative $\mathrm{VO}_{2 \max }$. Finally, consequently, the resistance group was also better at the walking performance (shorter time) as compared before the intervention (intragroup) $(p<0.0001)$ and with strength group $(p=0.000)$ and control group $(\mathrm{p}<0.0001)$ groups. But for this analysis, an improvement in time was also observed for the strength group $(\mathrm{p}=0.027)$. However, as the resistance group obtained better results in $\mathrm{VO}_{2 \max }$, these performance findings are compatible, and the individuals in this group had a higher $\mathrm{O}_{2}$ supply during the activity and, therefore, presented better performance.

The possible mechanisms related to hemodynamic and cardiorespiratory changes may be related to hormonal, neural, metabolic, and structural changes ${ }^{11}$. Regarding hemodynamics (heart rate and blood pressure), better neuro cardiac adaptations, and the renin-angiotensin blood pressure system may be the main factors of positive changes in this system. Additionally, reduced arterial stiffness, endothelial function, and plasma volume are also responsive in improving cardiovascular behavior ${ }^{22}$. In $\mathrm{VO}_{2 \max }$, improved transport and delivery of $\mathrm{O}_{2}$ to tissues due to higher hemoglobins and myoglobins are part of the chronic improvement of $\mathrm{VO}_{2 \max }{ }^{23}$. Another physiological mechanism that directly influences cardiovascular and respiratory responses is mitochondrial density, increasing the muscle cell's efficiency in absorbing and consuming $\mathrm{O}_{2}{ }^{24}$. These changes occur, perhaps, with greater magnitude in aerobic activities, but in resistance training, these changes are also plausible, and regardless of the type of activity, the mechanisms to be activated for possible cardiovascular and respiratory improvements are the same.

\section{Conclusions}

Through this study, it is possible to affirm that further studies to investigate hemodynamic and cardiorespiratory actions in the face of resistance training are necessary. This type of training and promoting neuromuscular improvements can also be an excellent strategy for improving the cardiovascular and cardiorespiratory systems of the elderly. Thus, it is indispensable in the regular training program for this population.

\section{Methods}

The study included 105 individuals (64 women and 41 men), physically active (equal to or more than 150 minutes per week), all over 60 years of age (Table 1). As an inclusion criterion, it was considered that the individual was fit for experimental intervention without any osteoarticular restriction, be independent in the performance of daily activities, and could not have been doing any (regular) physical 
exercise for at least three months. On the other hand, as an exclusion criterion, the multidisciplinary team considered any possible intervention restriction. Existing musculoskeletal injuries and the use of drugs to control and/or balance the cardiovascular system for psychological or neurological treatments, among others, were also reasons for exclusion from the research. Finally, other factors that could negatively affect the intervention results, such as morbid obesity and chronic kidney disease, were considered exclusion criteria.

A pilot study was used to determine the sample size. After calculating the sample size, it was found that each sample group should consist of at least 31 individuals.

The present study met the standards for researching human beings, resolution 196/96, the National Health Council of 10/10/1996 (Ministry of Health), and the Helsinki Resolution ${ }^{25}$. All study participants agreed to sign the informed consent form containing the study objective, assessment procedures, voluntary nature of the subject's participation. An information term was also prepared for the institution where the research was carried out, with the same items as the informed consent form and the questionnaire for physical activity readiness (PAR-Q). The study had its research project submitted to the Research Ethics Committee Involving Human Beings at Castelo Branco University, approved under nº 0013/2008.

\section{Experimental Design}

After the entire selection process, individuals were randomized to compose three groups, namely: Strength Group ( $\mathrm{n}=35)$, Resistance Group $(\mathrm{n}=35)$, and Control Group $(\mathrm{n}=35)$. Before the start of training, all individuals made three visits to the laboratory. The first was familiarization with all procedures, signing and filling out documents (informed consent form and PAR-Q), hemodynamic, and cardiopulmonary evaluation. The second and third visit (48-hour interval) was for the test and retest of $1 \mathrm{RM}$ to outline the loads to be applied in the training program exercises. The training was soon started and conducted for 16 weeks (Figure 4), with two weekly sessions, respecting a minimum of 48 and a maximum of 72 hours of an interval between sessions. The alternating method by segment was determined for training sessions, and the following exercises were selected: bench press, simultaneous squat, one-sided curved stroke, seated knee extension, simultaneous standing biceps, standing knee flexion, and triceps on the bench simultaneously. All exercises were performed freely (without the apparatus's aid), using short barbells, washers, and shin pads as accessories.

The control group maintained their routine daily chores throughout the study period. This group undertook not to carry out any systematic physical activity during the 16 weeks. The experimental groups performed the intervention until the post-test was carried out and completed the same evaluations as the experimental groups in the same period, thus considering pre and post. For the experimental groups, the training sessions followed the periodic adaptation (4 weeks) and specific (12 weeks) phases ${ }^{26}$.

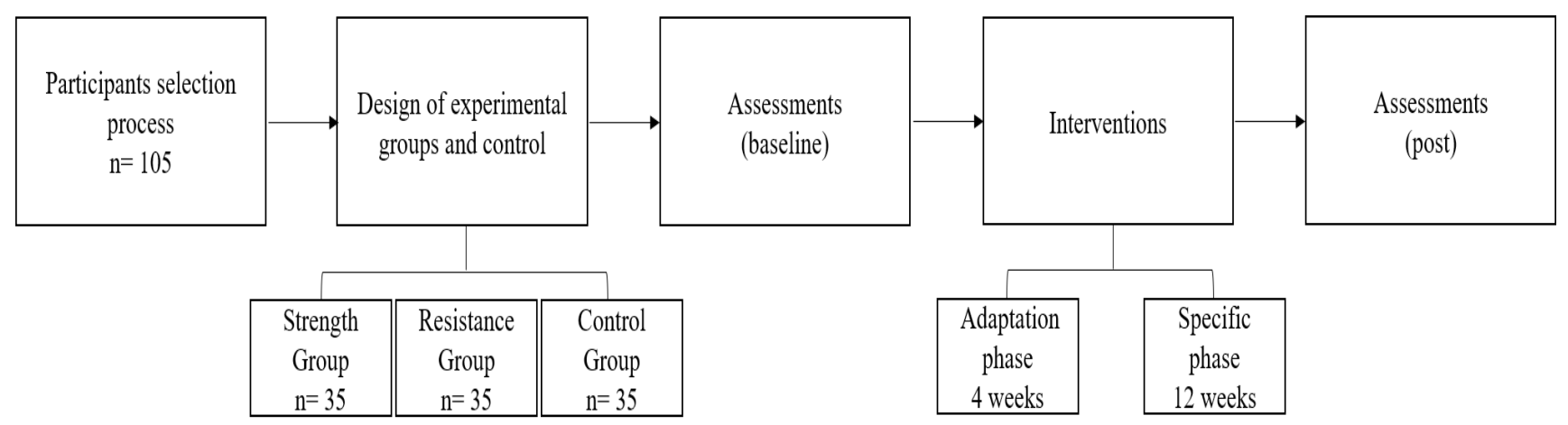

Figure 4. Flowchart of experimental design.

Both experimental groups (Strength Group and RG) performed four weeks of adaptation and 12 weeks of specific training. The Strength Group and the resistance group did four weeks adaptation phase 
and 12 weeks intervention load with $70-85 \%$ and $50-70 \%$, respectively. The same exercise program was used in the experimental groups, using the alternating method by segment with the bench press, squat, one-sided curved stroke, leg extension, biceps curl, knee flexion, Triceps forehead. Both the Strength Group and resistance group carried out two weekly sessions with an interval of at least 48 and a maximum of 72 hours.

Both phases had ten minutes of warm-up consisting of exercises that involved the mobility of the main joints. At the end of each session, stretching exercises were performed for muscle relaxation lasting five minutes ${ }^{27}$. The passive breathing used was elective, expiring in the concentric phase of the movement and inspiring in the eccentric phase, as it is the most suitable for this age group. Each group performed a layout of series and repetitions, interval time, speed of execution, and workload (Figure 5). The control group performed only daily activities (without excessive effort) and evaluations in the same experimental groups.

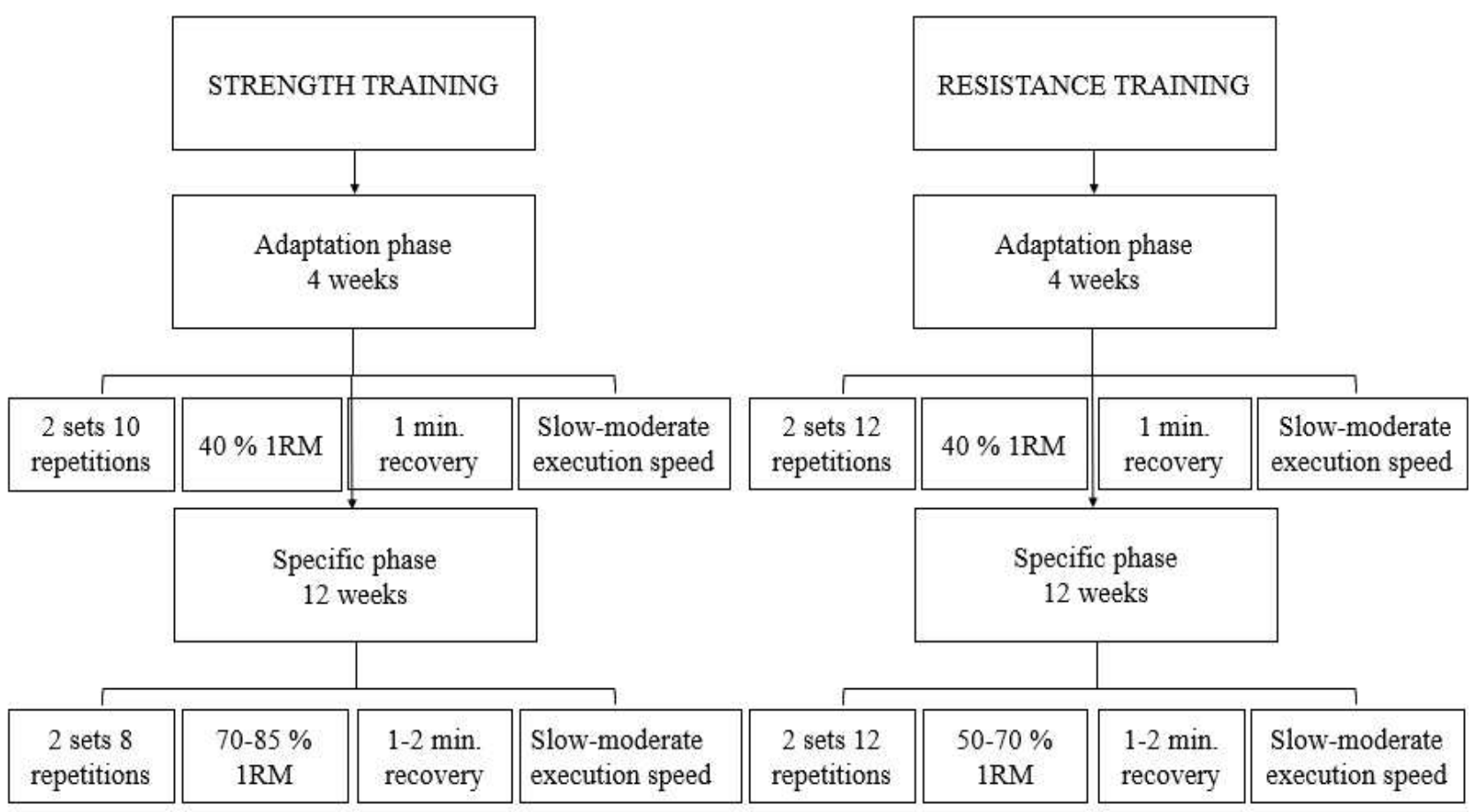

Figure 5. Schematic design of experimental protocols

\section{Hemodynamic Analyses}

The variables heart rate (HR) and blood pressure (systolic and diastolic) were used for hemodynamic analysis. Also, the rate pressure product was transferred using the equation: [HR (bpm) $\mathrm{x}$ SBP $(\mathrm{mm} / \mathrm{Hg})]^{28}$. For HR collection, a POLAR RS800CX ${ }^{\circledR}$ watch (Multisport ${ }^{\mathrm{TM}}$ model), Kempele, Finland ${ }^{29}$, was used. To assess systolic and diastolic blood pressure (SBP and DBP, respectively), a digital oscillometer device from the brand OMRON M6 (HEM-7001- E) ${ }^{\circledR}$ was used ${ }^{30}$. Individuals will be seated for 5 minutes of rest with the arms extended and with the arm placed at the heart level ${ }^{31}$.

\section{Maximum Oxigen Uptake Analyses $\left(\mathrm{VO}_{2 \max }\right)$ and walking performance}

The cardiorespiratory analysis was performed using the 1600 -meter test ${ }^{32}$, one of the most recommended assessments in the elderly population ${ }^{33}$. The test was carried out on a soccer field with official measures, and the objective was for the participants to complete the course in the shortest possible time. HR was collected immediately after the test. The time was measured using an OREGON® 
stopwatch (model C510-B ${ }^{\text {TM}}$ ) was used, and for HR collection, a POLAR RS800CX® ${ }^{\circledR}$ watch (Multisport model $^{\mathrm{TM}}$ ), Kempele, Finland ${ }^{29}$. After collecting the variables (time and heart rate), the equation below was used to calculate and the estimated value of $\mathrm{VO}_{2 \max }$. The duration of the activity was performed to assess the performance of the walk.

Equation:

$\mathrm{VO}_{2 \max }=132,853-(0,0769 x B o d y w e i g h t)-(0,3877 x a g e)+(6,315$ x gender $)-(3,2649 \times$ time $)-(0,01565 \times \mathrm{HR})$ where: lb $(1 \mathrm{lb}=0,454)$; Gender: female $=0$ / male $=1$; Time: minutes; HR: Collecting after test

The statistical analyses were carried out using the GraphPrism software version 8.0.1 with a significance level of $5 \%(\mathrm{p}<0.05)$. The Shapiro Wilk test was applied to test the normality of the data. The Test $\mathrm{T}$ was used for comparisons pre (baseline) and post-intervention in anthropometric data. For analysis of the investigation data, the two-way ANOVA of repeated measures (group x moment) was used, followed by Tukey's test for multiple comparisons, when necessary.

\section{References}

1. WHO. World health statistics 2016: monitoring health for the SDGs sustainable development goals. (World Health Organization, 2016).

2. Chang, E.-S. et al. Global reach of ageism on older persons' health: A systematic review. PLoS One 15, e0220857 (2020).

3. Chodzko-Zajko, W. J. et al. Exercise and physical activity for older adults. Med. Sci. Sport. Exerc. 41, 1510-1530 (2009).

4. Sant'Ana, L. de O. et al. Effects of Cardiovascular Interval Training in Healthy Elderly Subjects: A Systematic Review. Front. Physiol. 11, 739 (2020).

5. Fraga, M. J., Cader, S. A., Ferreira, M. A., Giani, T. S. \& Dantas, E. H. M. Aerobic resistance, functional autonomy and quality of life (QoL) of elderly women impacted by a recreation and walking program. Arch. Gerontol. Geriatr. 52, e40-e43 (2011).

6. Hoefelmann, C. P. et al. Aptidão funcional de mulheres idosas ativas com 80 anos ou mais Functional fitness of elderly active women aged 80 or more. Mot. Rev. Educ. Fis. 17, 19-25 (2011).

7. Ferreira, O. G. L., Maciel, S. C., Costa, S. M. G., Silva, A. O. \& Moreira, M. Envelhecimento ativo e sua relação com a independência funcional. Texto Context. enferm 21, 513-518 (2012).

8. Cipriani, NCS.; Meurer, ST; Benedetti, TRB; Lopes, M. Aptidão Funcional de Idosas Praticantes de Atividades Físicas. Rev. Bras. Cineantropometria E Desempenho Hum. 12, 106-111 (2010).

9. Fletcher, G. F. et al. Promoting Physical Activity and Exercise: JACC Health Promotion Series. Journal of the American College of Cardiology vol. 72 1622-1639 (2018).

10. SCARTONI, F. et al. Physical Conditioning Index on Active Elderly Population. Biomed. J. Sci. Tech. Res. 11, 1-4 (2018).

11. de Oliveira Sant'Ana, L. et al. Comparação das variáveis cardiovasculares em idosos ativos em diferentes modalidades físicas. Rev. Bras. Fisiol. do Exerc. 18, 186-194 (2020).

12. dos Reis, N. R., Vianna, J. M., Colugnati, F. B., da Silva Novaes, J. \& Mansur, H. N. Sensitivity and specificity of SARC-F in the classification of sarcopenia in the elderly: preliminary results. Rev. Bras. Fisiol. do Exerc. 19, 258-266 (2020).

13. Nelson, M. E. et al. Physical activity and public health in older adults: recommendation from the American College of Sports Medicine and the American Heart Association. Circulation 116, 1094 
(2007).

14. Garber, C. E. et al. Quantity and Quality of Exercise for Developing and Maintaining Cardiorespiratory, Musculoskeletal, and Neuromotor Fitness in Apparently Healthy Adults. Med. Sci. Sport. Exerc. 43, 1334-1359 (2011).

15. Fragala, M. S. et al. Resistance training for older adults: a position statement from the national strength and conditioning association. J. Strength Cond. Res. 33, (2019).

16. Pescatello, L. S. et al. Exercise, and Hypertension-ACSM Position Stand. Med. Sci. Sports Exerc. (2004).

17. Mota, M. R. et al. Acute and chronic effects of resistive exercise on blood pressure in hypertensive elderly women. J. Strength Cond. Res. 27, 3475-3480 (2013).

18. da Cunha Nascimento, D. et al. Sustained effect of resistance training on blood pressure and handgrip strength following a detraining period in elderly hypertensive women: a pilot study. Clin. Interv. Aging 9, 219 (2014).

19. da Cunha Nascimento, D. et al. Blood pressure response to resistance training in hypertensive and normotensive older women. Clin. Interv. Aging 13, 541 (2018).

20. Oliveira, N. A. de et al. Assessment of cardiorespiratory fitness using a submaximal protocol in older adults with a mood disorder and Parkinson's disease. Arch. Clin. Psychiatry (São Paulo) 40, 88-92 (2013).

21. Vianna, J. M., Werneck, F. Z., Coelho, E. F., Damasceno, V. O. \& Reis, V. M. Oxygen uptake and heart rate kinetics after different types of resistance exercise. J. Hum. Kinet. 42, 235-244 (2014).

22. Adamson, S. et al. Extremely short duration sprint interval training improves vascular health in older adults. Sports Sci. Health 15, 123-131 (2019).

23. Nemoto, K., Gen-no, H., Masuki, S., Okazaki, K. \& Nose, H. Effects of high-intensity interval walking training on physical fitness and blood pressure in middle-aged and older people. In Mayo Clinic Proceedings vol. 82 803-811 (Elsevier, 2007).

24. MacInnis, M. J. \& Gibala, M. J. Physiological adaptations to interval training and the role of exercise intensity. J. Physiol. 595, 2915-2930 (2017).

25. Helsinki, D. World Medical Association Declaration of Helsinki. Ethical principles for medical research involving human subjects. JAMA 284, 3043-3045 (2000).

26. Bompa, T. O. \& Buzzichelli, C. Periodization-: theory and methodology of training. (Human kinetics, 2018).

27. Dantas, E. H. M. A prática da preparação física (6a. ed.). (Grupo Gen - Editora Roca Ltda, 2014).

28. Ansari, M. et al. The association of rate pressure product (RPP) and myocardial perfusion imaging (MPI) findings: a preliminary study. Perfusion 27, 207-213 (2012).

29. Quintana, D. S., Heathers, J. A. J. \& Kemp, A. H. On the validity of using the Polar RS800 heart rate monitor for heart rate variability research. Eur. J. Appl. Physiol. 112, 4179-4180 (2012).

30. Topouchian, J. A., El Assaad, M. A., Orobinskaia, L. V, El Feghali, R. N. \& Asmar, R. G. Validation of two automatic devices for self-measurement of blood pressure according to the International Protocol of the European Society of Hypertension: the Omron M6 (HEM-7001-E) and the Omron R7 (HEM 637-IT). Blood Press. Monit. 11, 165-171 (2006).

31. Chobanian, A. V. National heart, lung, and blood institute joint national committee on prevention, detection, evaluation, and treatment of high blood pressure; national high blood pressure education 
program coordinating committee: the seventh report of the joint national. Jama 289, 2560-2572 (2003).

32. Kline, G. M. et al. Estimation of VO2maxfrom a one-mile track walk, gender, age, and body weight. Med. Sci. Sports Exerc. 19, 253-259 (1987).

33. Vale, R. G. S., Pernambuco, C. S. \& Dantas, E. H. M. Manual de avaliação do idoso. (2016).

\section{Consent for publication}

All participants were informed about the protocol and gave their written informed consent before participating in the study.

Availability of data and materials

The datasets analyzed during the current study available from the corresponding author [SCARTONI, FR] on the immediate request.

Funding

None

\section{Conflict of interest}

The authors declare no conflict of interest, financial or otherwise. 

Figures

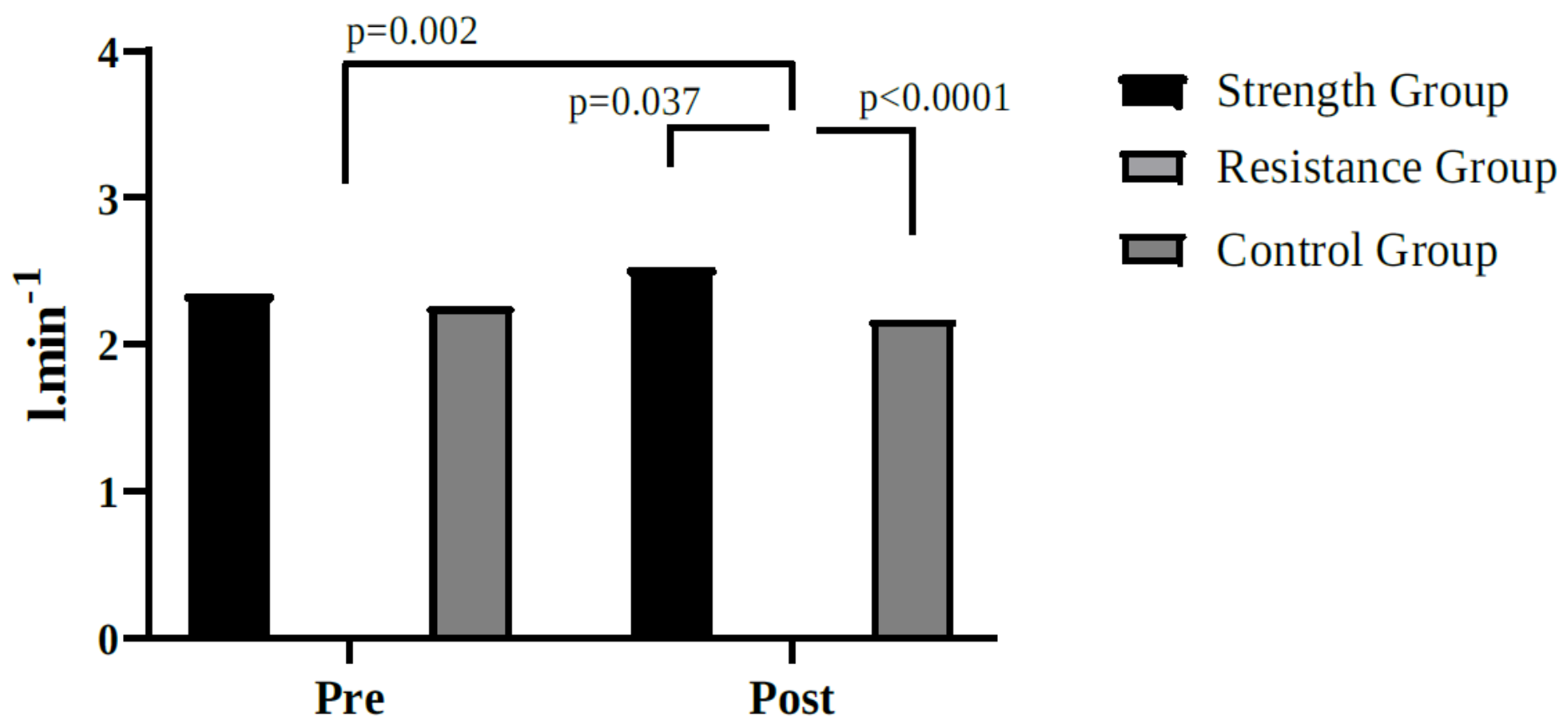

Figure 1

V02max absolute for the groups, before and after the intervention.

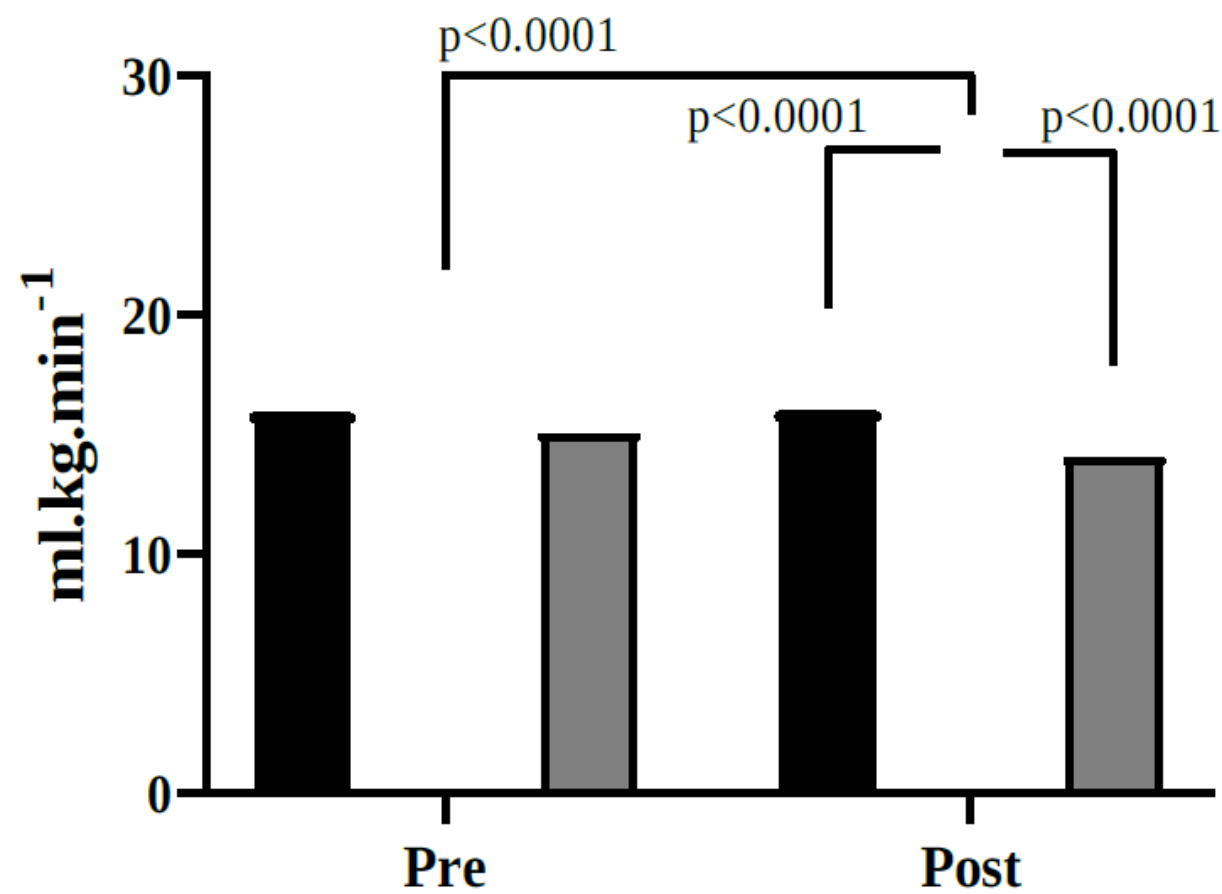

- Strength Group

$\square$ Resistance Group

$\square$ Control Group

Figure 2

Relative V02max for the groups, before and after the intervention. 


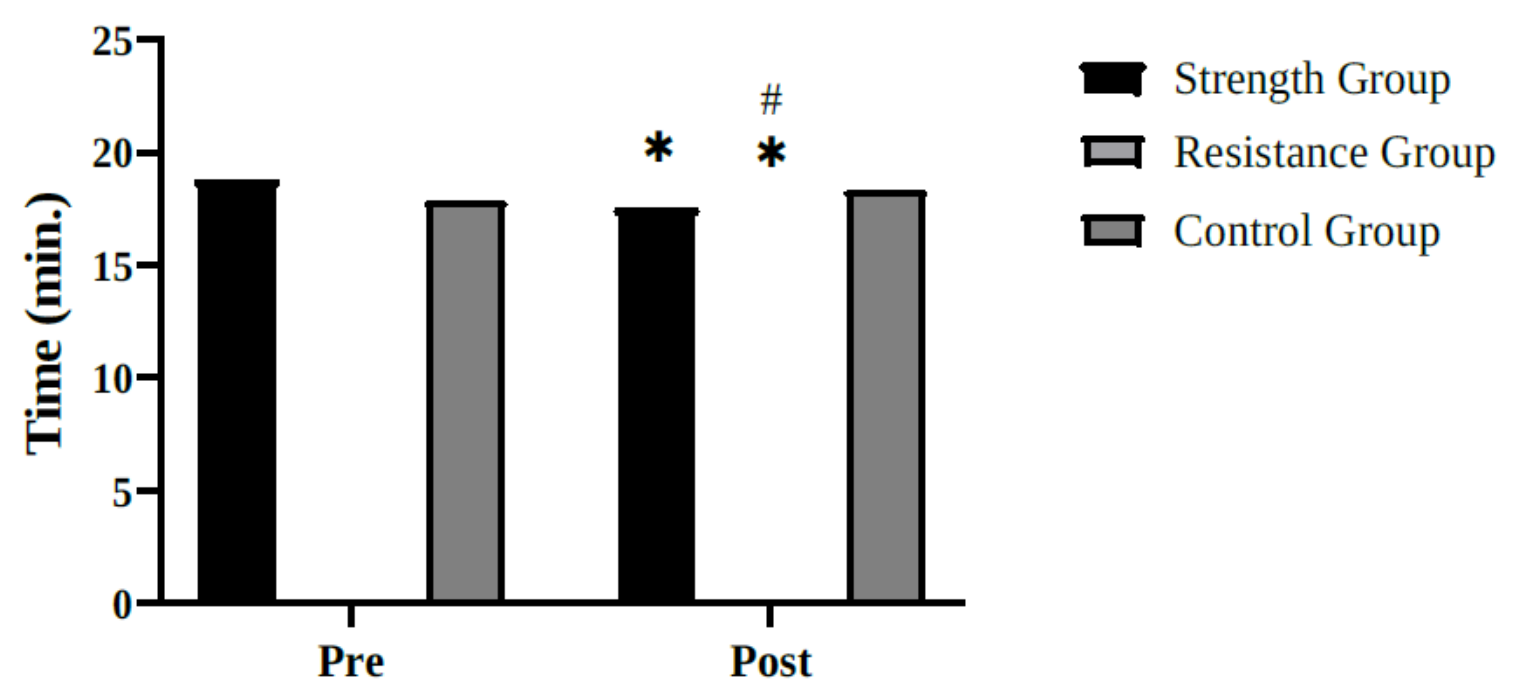

* Significant difference compared to the pre-intervention moment, for the same group ( $\mathrm{p}<0.05)$. \# Significant difference compared to the strength and control groups, after intervention $(\mathrm{p}<0.05)$.

\section{Figure 3}

Walking performance for groups, before and after interventions.

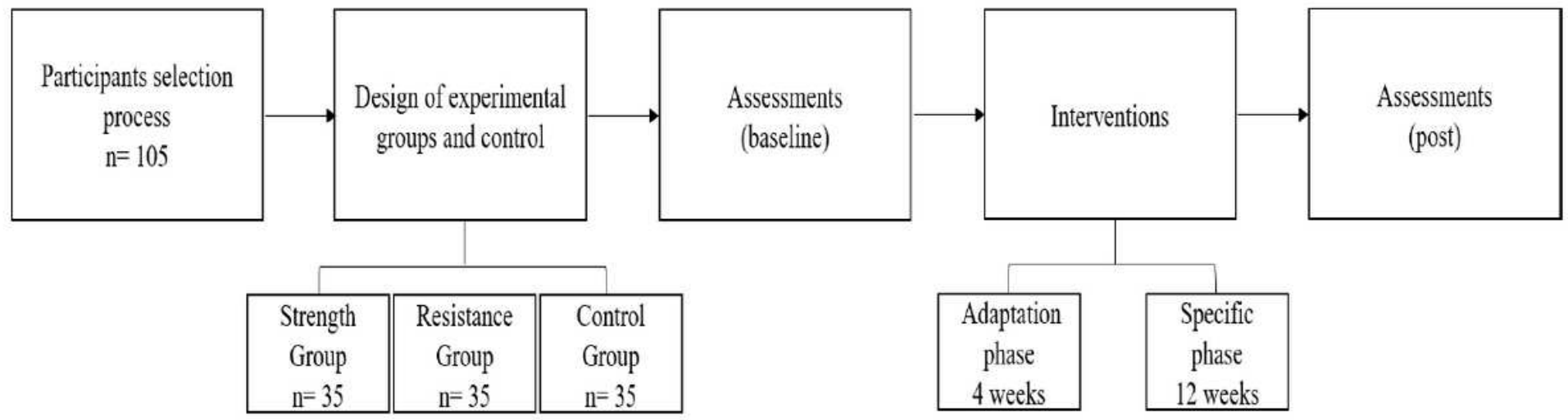

Figure 4

Flowchart of experimental design. 


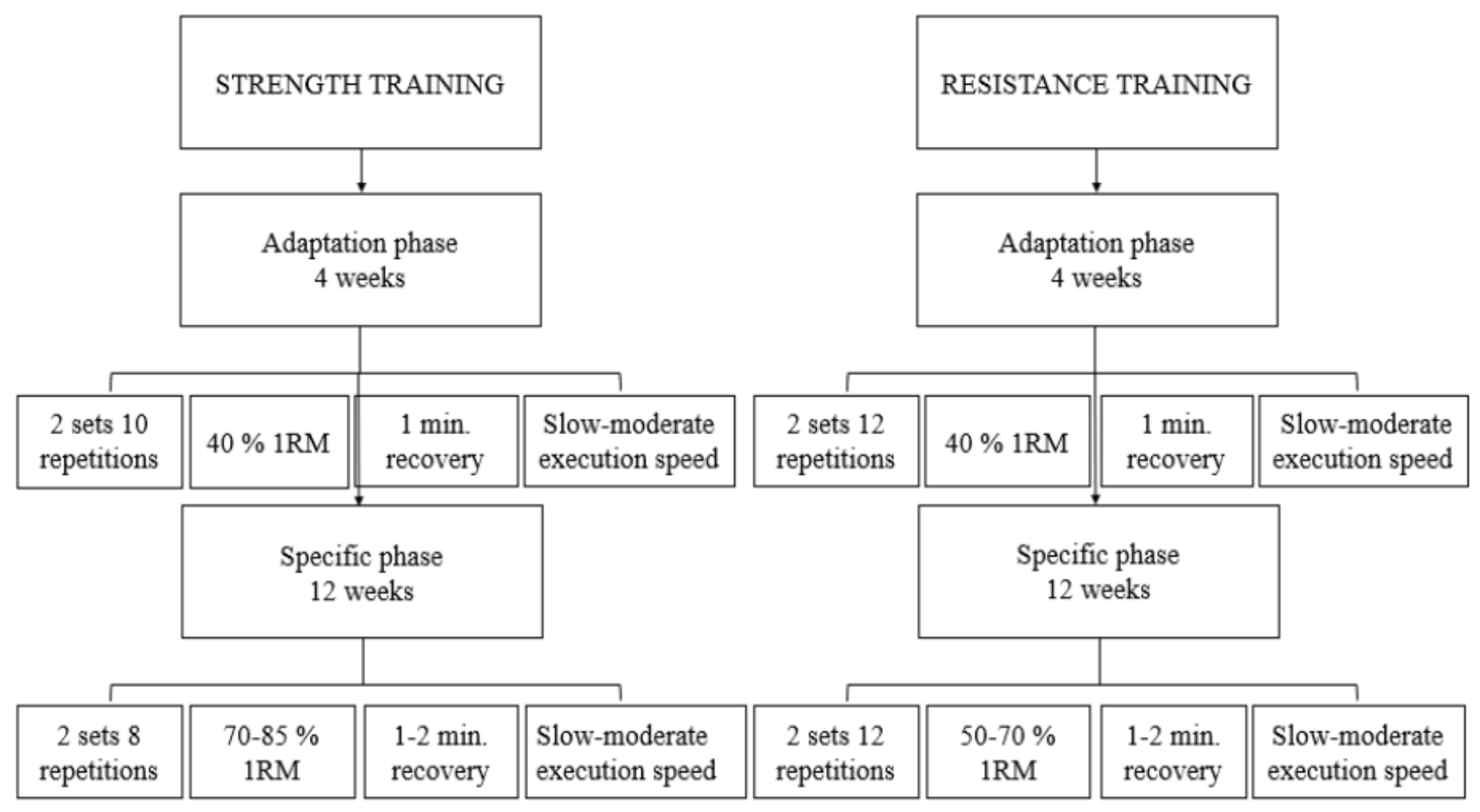

Figure 5

Schematic design of experimental protocols 